\title{
ENFERMEDAD DE TAKAYASU: A PROPÓSITO DE UN CASO
}

\author{
GUILLERMO OLAYA VILLARREAL ${ }^{1 *}$ \\ ${ }^{1 *}$ Médico Universidad del Quindío, Armenia Colombia \\ Residente de Medicina Interna, Universidad de Cartagena \\ “Correspondencia: Guillermo Olaya Villarreal.guillemedicine@gmail.com
}

Recibido: Enero 13 de 2015 Aceptado: Mayo 29 de 2015

\begin{abstract}
Resumen
Presentamos un caso de una paciente de 58 años, con enfermedad de Takayasu, también conocida como enfermedad sin pulsos, dicha patología es una enfermedad infrecuente, inflamatoria, granulomatosa vascular y de etiología desconocida que afecta a la aorta y a sus ramas principales, incluyendo la arteria carótida, así como la arteria subclavia, vertebral, renal y coronarias. Afecta a una de cada 200.000 personas. Las mujeres con esta enfermedad superan en número a los hombres por 8 a 1 , y la edad de comienzo se encuentra entre los 15 y los 30 años, siendo una de las causas de enfermedad cardiovascular temprana, siguiendo su proceso evolutivo de la enfermedad para terminar en la formación de aneurismas. Su tratamiento es a base de corticoides e inmunosupresores, aunque no hay suficiente evidencia que permita afirmar cual es mejor, en cuanto a eficacia y seguridad, la mayoría de veces queda relegado a criterio del facultativo, sobre todo si hay clara evidencia de la enfermedad.
\end{abstract}

Palabras clave: Arteritis de Takayasu, inflamación, vasculopatía, inmunosupresores, enfermedad sin pulso.

\section{TAKAYASU DISEASE: A CASE REPORT}

\begin{abstract}
We present a case of a 58 years with Takayasu disease, or pulseless disease, this illness is a rare disease, inflammatory, vascular granulomatous of unknown etiology that affects the aorta and its main branches, including the carotid artery, and subclavian artery, vertebral, renal and heart. It affects one in 200,000 people. Women with this disease outnumber men by $8-1$, and the age of onset is between 15 and 30 years, being one of the causes of early cardiovascular disease, following the evolutionary process of the disease to end in the formation of aneurysms. Treatment is with corticosteroids and immunosuppressants, although there is insufficient evidence that allows us to affirm what is best in terms of efficacy and safety, most often relegated to the physician's secrecy, especially if there is clear evidence of the illness.
\end{abstract}

Keywords: Takayasu arteritis, inflammation, vascular disease, immunosuppressive, Pulseless Disease. 


\title{
DOENÇA DE TAKAYASU: RELATO DE UM CASO
}

\begin{abstract}
Resumo
Apresentamos um caso de uma paciente de 58 anos com arterite de Takayasu, também chamada de doença sem pulso, esta doença é uma doença rara, inflamatória, granulomatosa vascular e de etiologia desconhecida que afeta a aorta e seus principais ramos, incluindo a artéria carótida assim como a subclávia, vertebral, renal e coronária. Ela afeta uma em 200.000 pessoas. As mulheres com esta doença superam os homens por 8 a 1. A idade do início situa-se entre 15 e 30 anos, sendo uma das causas da doença cardiovascular precoce, na sequência da sua evolução da doença para terminar na formação de aneurisma. O tratamento é baseado nos corticóides e imunossupressores, ainda não há provas suficientes para dizer qual é melhor, em termos de eficácia e segurança, na maioria das vezes é relegado ao critério do médico, especialmente se houver uma clara evidência da doença.
\end{abstract}

Palavras-chave: Takayasu, inflamação, doença vascular, imunossupressoras, doença sem pulso.

\section{Introducción}

La enfermedad de Takayasu es una patología crónica, que corresponde a las vasculitis de grandes vasos, y del grupo de las formas granulomatosas, es más frecuente en menores de 40 años (1). Es la tercera vasculitis más frecuente de la infancia, El primer caso de arteritis de Takayasu fue descrito en 1908 por el doctor Mikito Takayasu (2), el describió un caso con cambios peculiares de los vasos retinales centrales. Dos médicos japoneses (Dr. Onishi y Dr. Kagoshima) encontraron anomalías similares en los ojos de pacientes cuyo pulso estaba ausente, y en 1952 Caccamise and Whitman reportaron el primer caso (3), en 1960 Contorni publicó el primer reporte de flujo retrogrado, en una arteria vertebral asociado a oclusión de la arteria subclavia. La arteritis de takayasu afecta principalmente grandes vasos como la aorta y sus ramas principales, así como las coronarias y arterias pulmonares. La inflamación y subsecuente proliferación de la íntima causan lesiones estenóticas $\mathrm{u}$ oclusivas, mientras que las lesiones que causan destrucción de las capas elástica y muscular causan dilataciones vasculares o aneurismas. Aunque su distribución es mundial, es más frecuente en Japón, Asia, África y Latino-América que en Norte América y Europa. Del 80 a $90 \%$ de los pacientes son de género femenino, sobretodo en etapa reproductiva (4) con edad de inicio de la enfermedad entre los 10 y 40 años, más frecuente en asiáticos (5), induce una variedad de síntomas clínicos debidos a la isquemia causada por la lesión estenótica, aneurismática u oclusiva de la arteria afectada, por destrucción progresiva de la capa media o por formación del trombo. Los síntomas clínicos son: mareo, sincope, alteración de la visión, disminución o ausencia de pulso, soplos de hipoaflujo. La apoplejía, falla cardíaca o muerte por ruptura aneurismática pueden ser sus complicaciones más catastróficas. No existe una prueba de laboratorio diagnóstica específica de la enfermedad (6).

\section{Reporte de un caso}

Se trata de una paciente de género femenino de 58 años al momento de la consulta, con sintomatología desde hace 9 años atrás, procedente de Viterbo, Caldas, quien consulta por debilidad en piernas, síntomas de claudicación, astenia, adinamia, en la consulta externa, se evidencia ausencia de pulsos, en miembros superiores y pedio.

Al Examen físico: presión arterial 120/60 Frecuencia cardíaca 68 , frecuencia respiratoria 14 , peso $52 \mathrm{~kg}$ talla 1,53 cms, IMC: 22,21 afebril, disminución de pulsos en miembros superiores, y aortoiliaco, resto es normal, posteriormente soplo audible a nivel de mesogastrio así como ausencia de pulso en tibial posterior y pedio derecho ausentes. Arteriografía marzo del 2012 con oclusión de la arteria tibial anterior, la derecha con reconstitución distal y la izquierda no reconstituida, oclusión completa y corta de la arteria iliaca primitiva derecha, estenosis severa en origen de la arteria iliaca primitiva izquierda, posteriormente fue llevada a colocación de stent endoprotésico bilateral. 


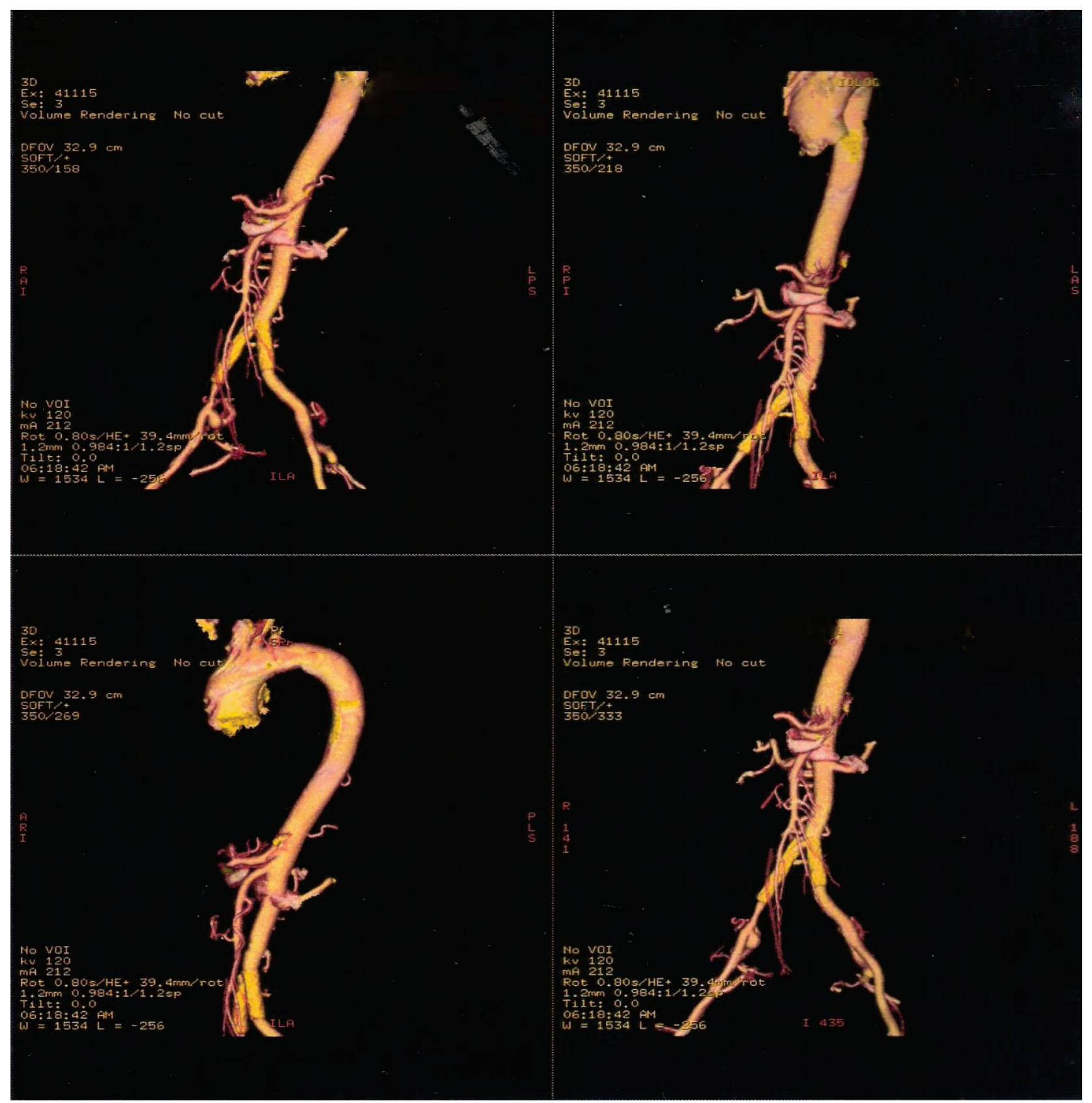

Figura 1. Lesiones estenóticas. Tomado del examen original del paciente, sin modificación.

Prosiguiendo con los exámenes tomados al paciente encontramos:

\section{Exámenes complementarios}

En el 2012 fue realizado cateterismo que evidencio obstrucción de arterias iliacas bilateral que requirió implantación de stent bilateral, arteriografía marzo del 2012 con oclusión de la arteria tibial anterior, la derecha con reconstitución distal y la izquierda no reconstituida, oclusión completa y corta de la arteria iliaca primitiva derecha, estenosis severa en origen de la arteria iliaca primitiva izquierda, posteriormente fue llevada a colocación de stent endoprotésico bilateral.

Al paciente se le llevo a cabo una angioresonancia de miembro superior (2013) con contraste, que mostro estenosis proximal de la subclavia izquierda con 
reconstitución distal, arteria braquial con disminución del calibre, estenosis múltiple en arteria vertebral izquierda, doppler de vasos de cuello (2013) que mostró oclusión de la arteria vertebral derecha, y arteriopatía de la región preescalénica de la subclavia izquierda con robo de la subclavia izquierda, se le practica angioresonancia de cuello con gadolinio (2014) concluyéndose estenosis crítica de la subclavia izquierda tanto a nivel prevertebral donde hay un segmento en donde no se observa flujo como en el postvertebral con estenosis crítica de más del 50\%. En la arteria subclavia derecha proximal al ostium de la vertebral sin evidencia de flujo, y distal zona de estenosis crítica postescalénica, estenosis de alto grado de la arteria vertebral derecha segmento v1, además sugiere flujo lento o invertido en la circulación posterior(dependiente del robo de la subclavia izquierda), angioresonancia cerebral simple (2014) demostró ausencia de flujo en segmento v4 de la arteria vertebral ni de la parte baja de la arteria basilar, arteriopatía compatible con Takayasu, por ausencia de flujo de la arteria vertebral, arteria basilar, y cerebelosas posteroinferiores, además se evidenció lesión en sustancia blanca (infarto frontoparietal izquierdo), no se evidenció aneurisma ni malformación arteriovenosa.
Como vemos el diagnóstico fue corroborado por la aplicación de los criterios clínicos y radiológicos del American College of Rheumatology (ACR), y clasificada esta paciente como una arteritis de takayasu tipo 5 de la clasificación de Numan.

Se inicia Metotrexate 20 mg semanal vía oral, además de ácido fólico, fue valorada por oftalmología quien solo evidencia presbicia.

Ecocardiograma doppler color modo m, bidimensional (2014) función sistólica conservada no hipertensión pulmonar PSAP $18 \mathrm{Mm} \mathrm{Hg}$ y no alteraciones cardiacas globales.

Posteriormente fue llevada a PET-CT oncológico 5-022014 que mostró aumento anormal del metabolismo en las paredes de vasos: cayado de la arteria carótida interna, emergencia del tronco braquiocefálico, la arteria subclavia izquierda tercio distal, cayado de la aorta, en relación con proceso inflamatorio activo tipo arteritis de takayasu, además aumento del metabolismo a nivel de la prótesis aortobifemoral izquierda.

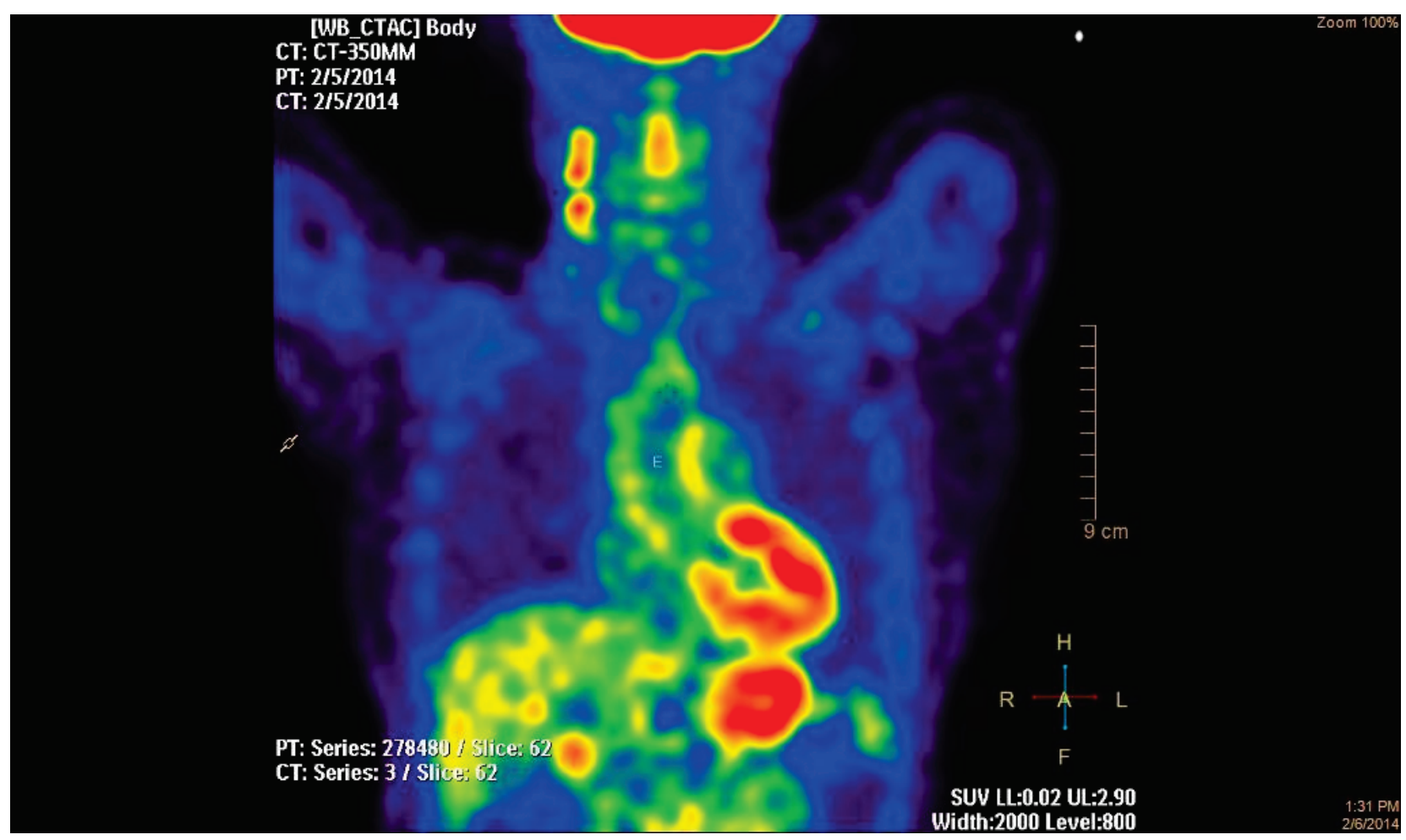

Figura 2. Metabolismo anormal en pet-ct oncológico. Tomado del examen original del paciente, sin modificación. 

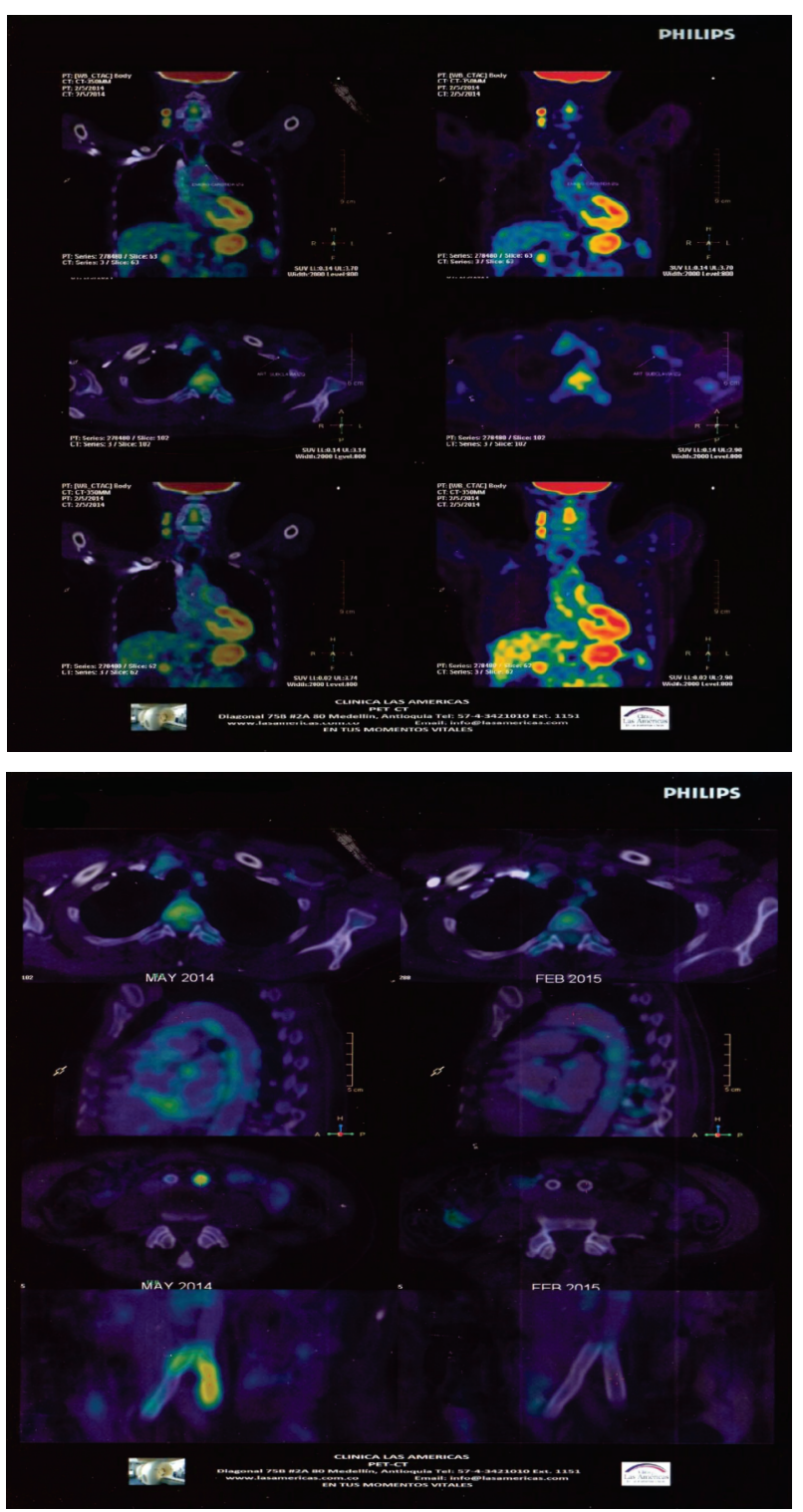

Figura 3 y 4. Estudio comparativo pet-ct oncológico 2015 vs 2014 en donde se ve disminución notable del metabolismo posterior al tratamiento con Metotrexate. Tomado del examen original del paciente.

Los exámenes de anticuerpos (2014) Anticardiolipina IGM $\mathrm{U}(0,001 \mathrm{MPL} / \mathrm{ml})$ e IGG U $(0,56 \mathrm{GLP} / \mathrm{ml})$, anticoagulante lupico $(1,13)$ y beta dos glicoproteína IGM e IGG $(0,20$ y $0,19 \mathrm{u} / \mathrm{ml}$ ) todos fueron negativos, es decir, se descartó la posibilidad de un síndrome anti-fosfolípidos, hemograma inicial normal, luego hemograma de control leucocitos $5320 \mathrm{~mm}^{3}$, hemoglobina $11,7 \mathrm{~g} / \mathrm{dL}$, hematocrito $37 \%$, plaquetas 158.000 , VSG $6 \mathrm{~mm} /$ hora) normal, PCR negativo, creatinina $(0,57 \mathrm{mg} / \mathrm{dl})$ normal.
Finalizando 2014 angioresonancia de cuello con contraste arteria subclavia izquierda con estenosis proximal y angioresonancia cerebral que solo mostro área de microangiopatía periventricular izquierda, demás vasos normales. Y doppler carotideo (2015) dentro de límites normales del lado derecho y robo de la subclavia del lado izquierdo, nuevo PET-CT para evaluación de respuesta al tratamiento reporta con respecto al estudio previo: desaparece el proceso inflamatorio activo a nivel de la endoprotesis ileofemoral, desaparece el proceso inflamatorio activo a nivel del tercio medio de la subclavia izquierda, es decir en relación con adecuada respuesta al tratamiento en estas regiones, además persiste pero disminuye de manera importante el proceso inflamatorio a nivel del tronco braquiocefálico, la carótida común izquierda y carótida interna izquierda, persiste sin aumento anormal en el metabolismo la obstrucción de la arteria subclavia derecha.

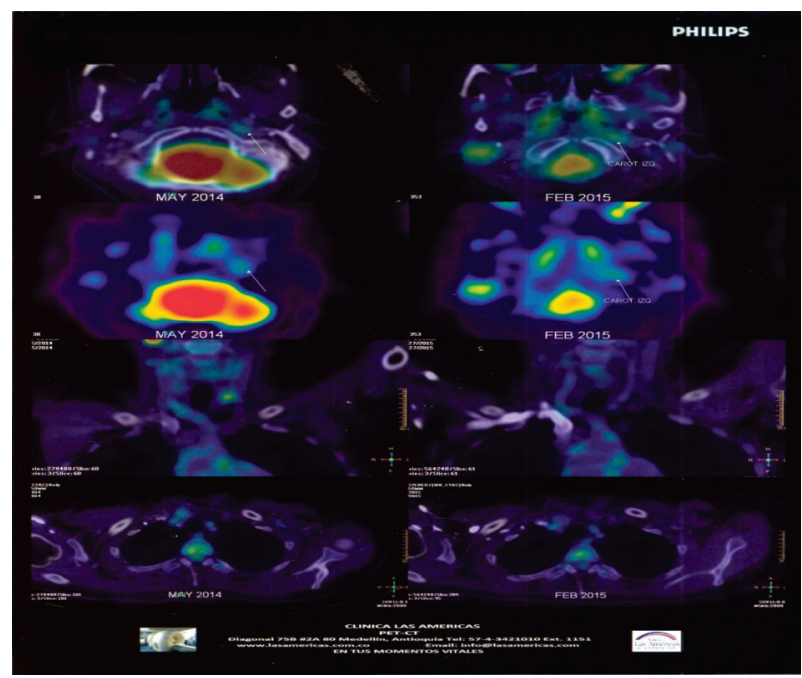

Figura 5. Notable disminución metabólica de la inflamación en un año de tratamiento. Estudio comparativo. Tomado del examen original del paciente.

\section{Discusión}

La arteritis de Takayasu o síndrome del Arco Aórtico, se ha descrito en relación con diferentes antígenos de Histocompatibilidad DR2 y MB1 en oriente y DR4 y MB3. En Norteamérica tiene una distribución mundial pero con una prevalencia mayor en Asia; en Japón su incidencia es de 150 casos nuevos por año. El 80\% al 90\% de los pacientes son mujeres, con una edad de inicio entre los 10 y los 40 años (1).

Es una enfermedad rara con una frecuencia anual de 1,2 a 2,6 casos por millón de habitantes (4). El estudio de Cañas 
y Col (7). Muestra que en Colombia la edad de inicio está entre los 13 y 47 años y que la edad de los pacientes al momento del diagnóstico fue 30.6 +/- 9.4 años, se encontró igualmente un predominio de la enfermedad en mujeres (relación 2.9:1) (7). Otro estudio realizado por Ochoa, Cañas y Col (8) mostró la epidemiología de las vasculitis primarias en Colombia y comparándolas con las de Latinoamérica, encontró que hay en Colombia 857 casos de vasculitis primaria. La arteritis de Takayasu fue la vasculitis más frecuente en un 13,3\% (114 casos) seguida de la enfermedad de Buerger en 11,2\% (96 casos), las vasculitis cutáneas primarias y la poliarteritis nodosa en un 10\% (86 casos) cada una.

En niños, la vasculitis más frecuente fue la púrpura de Henoch Schönlein en un 24\% (206 casos). En Latinoamérica se publicaron 177 artículos con 1605 casos informados. Se evidenció mayor presencia de arteritis de Takayasu en México y Brasil, y de poliangeitis microscópica en Chile y Perú. Colombia es el segundo País después de México en reporte y descripción de casos de vasculitis seguido por Brasil, chile, Perú. Esta vasculopatía también ha sido asociada a otras enfermedades de origen autoinmune, como las glomerulonefritis, aunque la enfermedad glomerular es excepcional; se han descrito algunos casos, e incluso estenosis de la arteria renal, con injuria renal que posteriormente empeora la progresión de la enfermedad de Takayasu.

Revisamos un caso de glomerulopatía del tipo mesangioproliferativa, documentada incluso en población pediátrica, en donde las glomerulopatías primarias son poco frecuentes, a excepción de la enfermedad de cambios mínimos, las demás, son con frecuencia de predominio en adultos: glomerulonefritis membranoproliferativa, glomerulonefritis membranosa, nefropatía por IGA, glomeruloesclerosis focal y segmentaria y la membranoproliferativa (ésta última documentada en un caso pediátrico (9-11), se observó también como causa de injuria renal asociado a arteritis, estenosis de la arteria renal causando hipertensión renovascular, 11 pacientes fueron seguidos por 155 meses observándose empeoramiento de la función renal en 7 , con muerte por causa renal asociada en dos casos (12).

\section{Patología}

La Inflamación activa es manifestada por la presencia de células mononucleares, especialmente histiocitos, linfocitos y macrófagos. Con la destrucción de la capa media muscular y la lámina elástica se pueden producir aneurismas. Con la progresión del proceso inflamatorio, la proliferación de la íntima contribuye al desarrollo de lesiones estenóticas. La arteritis de Takayasu se caracteriza por una panarteritis o inflamación de las tres túnicas de la arteri a (intima, media y adventicia) que inicia en esta última y progresa hacia la íntima (13).

\section{Clasificación}

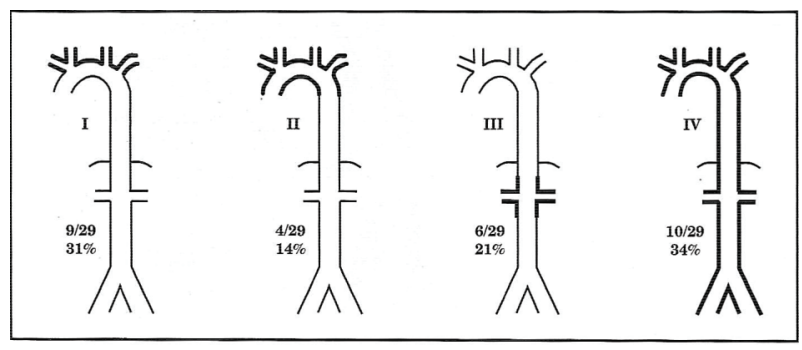

Figura 6. Hallazgos angiográficos según clasificación de Nasu. Tomado de Acta Medica Colombiana 1997, Vol. $22 N^{\circ} 2$

Anatómicamente, la arteritis de Takayasu se clasifica en cuatro tipos, según Nasu (7), el tipo I, se subdivide en IA Y IB.

- Tipo IA: es la más típica e involucra aorta ascendente, arco aórtico y sus ramas.

- Tipo IB: con localización similar a la IA, pero con dilatación aneurismática de la aorta torácica proximal.

- Tipo II: involucra la aorta torácica descendente y abdominal.

- Tipo III: es una combinación de tipo I y II.

- Tipo IV: involucra la arteria pulmonar, usualmente acompañado de aortitis $(7,14)$.

Según Numano, se clasifica:

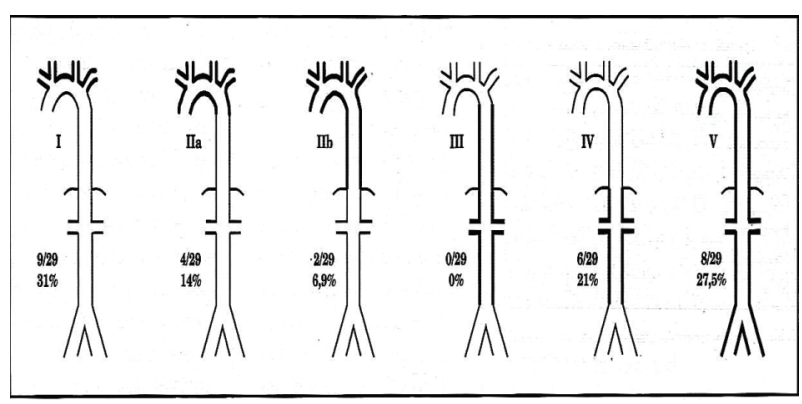

Figura 7. Hallazgos angiograficos según clasificación de Numano.

- Tipo I: compromiso de las ramas del cayado aórtico.

- Tipo Ila: aorta ascendente, arco aórtico y sus ramas.

- Tipo Ilb: aorta ascendente, arco aórtico, sus ramas y aorta torácica descendente.

- Tipo III: aorta torácica descendente y abdominal.

- Tipo IV: aorta abdominal y/o compromiso de las renales y

- Tipo V(arco aórtico, aortas torácica y abdominal) 


\section{Etiología}

Aunque no tiene una causa específica, es de etiología desconocida, aunque existen algunas evidencias que sugieren patogenicidad autoinmune con mecanismos humorales y celulares.

\section{Distribución}

La enfermedad puede localizarse en una porción de la aorta abdominal torácica, abdominal y sus ramas, o involucrar a toda la aorta. En orden de frecuencia se pueden afectar las arterias subclavias (85\%), aorta torácica descendente (58\%), abdominal (20\%), vertebral (17\%), ilíaca (16\%), pulmonar (15\%) y coronarias, en menos del $10 \%(15,16)$. La afectación de la aorta abdominal y de las arterias renales uni o bilateralmente es común, lo que puede llevar a hipertensión arterial e isquemia renal. En adultos se describe entre un 33 a $83 \%$ de hipertensión y la mayoría de las publicaciones pediátricas muestran a la hipertensión como una de las características principales. El compromiso de las arterias carótidas comunes puede provocar isquemia e infartos cerebrales (13).

\section{Cuadro clínico}

La enfermedad tiene dos fases distinguibles, una fase aguda y una fase crónica. En la fase aguda el paciente presenta síntomas generales que se relacionan con la severidad de la inflamación vascular como fiebre prolongada, malestar general, pérdida de peso, mialgias, elevación de reactantes de fase aguda, trombocitosis que representan el efecto de la citoquinas, particularmente interleuquina 6 , producida en las áreas de inflamación aguda.

A medida que progresa la inflamación vascular se presentan cambios irreversibles de la pared vascular como estenosis en la mayoría de los casos, aneurismas o cambios mixtos; estos cambios se manifiestan como síntomas explicables por insuficiencia vascular y circulatoria como claudicación de las extremidades, soplos carotideos o disminución de los pulsos en las extremidades, particularmente las superiores con la afectación de las arterias subclavias, tronco braquiocefálico, femorales o iliacas, el compromiso de las arterias renales resulta en hipertensión arterial. Los síntomas se relacionan con el territorio vascular afectado aunque es una patología de sospechar en pacientes jóvenes, así mismo es importante tener en cuenta en ausencia de pulsos en recién nacido, pensar y sospechar que esta patología puede verse incluso en periodo neonatal (17).

En la arteritis de Takayasu los síntomas neurológicos se presentan en más de la mitad de los pacientes y se producen como consecuencia de la estenosis de las arterias carótidas y/o vertebrales. Las manifestaciones más frecuentes son cefalea, vértigo, inestabilidad, síntomas ortostáticos y síncope. Otras complicaciones más graves, como accidente cerebrovascular isquémicos ocurren en $5 \%$ de los pacientes. Las alteraciones visuales, como la amaurosis fugaz o pérdida de visión permanente se producen en 10\% de los casos.

Otra causa importante de disminución o pérdida de la agudeza visual, sobre todo en pacientes de raza asiática, es la retinopatía hipotensiva, producida por la hipoperfusión de los vasos retinianos y coroidales, secundaria a la estenosis de las arterias carótidas (18).

El diagnóstico de estas enfermedades suele retrasarse debido a lo inespecífico de los síntomas y a que no existen marcadores de laboratorio fiables que permitan valorar la actividad de la enfermedad; tampoco se han validado adecuadamente las diferentes técnicas de imagen disponibles actualmente, sobre todo arteriografía (19) que es el patrón Gold standar y la angioresonancia $(19,20)$.

\section{Criterios diagnósticos}

El diagnóstico de esta entidad se hace con base en los criterios del Colegio Americano de Reumatología publicados en 1990. Los criterios diagnósticos (21) propuestos por la Asociación Americana de Reumatología (ARA) en 1990 son seis:

- Claudicación intermitente, especialmente de extremidades superiores.

- Edad de comienzo de la enfermedad igual o menor de 40 años.

- Disminución del pulso de una o ambas arterias braquiales.

- Diferencia en la tensión arterial sistólica entre ambos brazos mayor de $10 \mathrm{Mm} \mathrm{Hg}$.

- Soplo audible a la auscultación sobre una o las dos arterias subclavias o la aorta abdominal.

- Arteriografía anormal, caracterizada por presencia de estenosis y dilataciones arteriales.

La presencia de tres o más de estos criterios demostró una sensibilidad diagnóstica del $90,5 \%$ con una especificidad del $97,8 \%$.

Otros criterios diagnósticos fueron definidos por Ishikawa (22), este establece criterios mayores y menores:

Mayores:

- Edad inferior a 40 años (obligatorio) y afectación de la arteria subclavia. 


\section{Menores:}

- Aumento de la velocidad de sedimentación

- Aumento de la presión arterial

- Dolor en el territorio de la arteria carótida, y lesiones arteriales ubicadas en: aorta, tronco braquiocefálico, arteria carótida primitiva, o arteria pulmonar. Se establece diagnóstico con dos criterios mayores o un criterio mayor y dos menores, o con cuatro criterios menores.

\section{Hallazgos de laboratorio}

Los hallazgos de laboratorios reflejan el proceso inflamatorio, pero no son específicos. En la mayoría de los pacientes se encuentra anemia de enfermedad crónica. Los reactantes de fase aguda como velocidad de sedimentación eritrocitaria, incremento de la proteína $C$ reactiva, autoanticuerpos asociados a otras formas de enfermedad vascular, incluyendo anticuerpos antinucleares, anticuerpos anticitoplasma de neutrófilos y anti-DNA y anticuerpos antifosfolípidos no son encontrados en arteritis de Takayasu.

\section{Diagnóstico diferencial}

Ante un paciente joven con clínica cerebrovascular se debe pensar además del Takayasu en enfermedades embolígenas, síndromes de hipercoagulabilidad, disección carotidea, vaso espasmo por cocaína. Tener en cuenta las asociaciones de la arteritis de Takayasu a otras patologías, sobre todo de origen autoinmune, su coexistencia y su superposición de síntomas también pueden ser clasificados inicialmente como diagnósticos erróneos y con la evolución llegar a un diagnóstico tardío de esta patología, en la literatura reciente hay casos destacables como por ejemplo un caso diagnosticado en edad pediátrica, inicialmente como fiebre reumática con alteración valvular mitral, teniendo evolución hacia una arteritis de Takayasu, siendo los hallazgos mitrales una complicación de la afectación sistémica endotelial de esta patología (23) . El diagnóstico se retarda aún más si los criterios para ello, pueden verse limitados en su utilidad, si se tiene en cuenta la falta de un grupo control con enfermedad aortica, sobre todo en edades pediátricas (24).

\section{Tratamiento}

Lamentablemente no hay estudios controlados para tratar de ponderar el efecto del tratamiento, la raza y otros factores sobre el pronóstico de estos pacientes. Cabe señalar que con el uso de glucocorticoides e inmunosupresores se alcanzan índices de remisión altos pero también hay una alta frecuencia de recaídas sobre todo al iniciar la disminución de las dosis de los glucocorticoides. Debido a que casi no hay estudios controlados sobre el tratamiento en las vasculitis de grandes vasos, no hay suficiente evidencia que permita conocer cuál es el mejor tratamiento en cuanto a eficacia y seguridad y la mejor estrategia para mantener la remisión y mejorar el pronóstico. En la mayoría de los casos el tratamiento se basa en el juicio del clínico. Recientemente el European League Against Rheumatism (EULAR) publicó recomendaciones para el manejo de vasculitis de grandes vasos basándose en una revisión sistemática de la literatura y un consenso de expertos. Son varias recomendaciones, las cuales tienen un nivel de evidencia bajo pues solo hay estudios descriptivos y por lo tanto los grados de recomendación no son mayores de $\mathrm{C}$. En general el uso de glucocorticoides $(G C)$ e inmunosupresores están indicados si hay una clara evidencia de actividad.

La gran mayoría de los pacientes responden a la prednisona. La dosis inicial normal es de un miligramo por kilogramo de masa corporal por día (40- 60 miligramos al día) (4). Debido a los fuertes efectos secundarios del uso a largo plazo de la prednisona, la dosis inicial es regulada a las pocas semanas por los médicos para que ésta sea tolerable por el paciente.

Ruige et al (25), utilizan corticoides y en caso de mala respuesta, azatioprina o Metotrexate (4), en dosis de hasta $25 \mathrm{mg}$ semanal, controlando la actividad de la enfermedad a través de la velocidad de eritrosedimentación, leucocitosis o signos clínicos de inflamación, pero se plantean si un uso más agresivo del tratamiento pudiera evitar complicaciones $e$ incluso disminuir las cifras de mortalidad, aunque el pronóstico depende del daño, extensión y ubicación de las lesiones. Mejor sobrevida se ha obtenido con el uso de inmunosupresores en combinación con corticoides en días alternos por tiempos prolongado $(26,27)$.

La literatura no es clara en cuanto al mejor tratamiento y especialmente al período de tiempo óptimo de tratamiento, incluso existen publicaciones, como la de Kerr et al. (26), en donde describen que la disminución de los corticoides, luego de recibir altas dosis, resultaba en exacerbaciones de la enfermedad. Park da importancia a la velocidad de eritrosedimentación como índice de actividad de la enfermedad $e$ incluso, debido a la dificultad de un buen seguimiento por imágenes, aconseja utilizar este examen como un marcador de actividad en la práctica clínica (28).

Un cuidado oportuno, eficiente e incluso agresivo en controlar la actividad de la enfermedad y especialmente las complicaciones, se ha demostrado de gran ayuda para mejorar el pronóstico a largo plazo de la Arteritis de Takayasu. El uso de inmunoglobulina intravenosa solo queda relegado a casos refractarios, no se apoya su uso de rutina (28). El pronóstico es favorable, el resultado a largo plazo varia con el estadio en dos estudios estadounidenses 
se encontró una supervivencia global del 94\%, si bien la mortalidad a 5 años en otros estudios varia hasta 35\%, esta mortalidad dependió de insuficiencia cardíaca, enfermedad vascular cerebral, infarto agudo de miocardio, rotura de aneurisma estenosis de la arteria renal, que evoluciona a insuficiencia renal. También se ha descrito evolución hacia glomerulonefritis y casos de asociación entre arteritis de Takayasu y fiebre reumática, así como enfermedad de Takayasu en recién nacidos.

\section{Conclusiones}

Presentamos el caso de una paciente con vasculopatía por Takayasu, enfermedad poco común, frecuente en el sexo femenino, de etiología desconocida, y que su diagnóstico no debe pasar desapercibido, porqué al ser un proceso crónico y destructivo con lleva a complicaciones y morbilidad significativa, dado que no hay pruebas específicas su diagnóstico se ve retrasado, hasta los estudios imagenológicas que demuestren estenosis o aneurismas, pero siempre debemos pensar en ella, sobre todo en patología con ausencia de pulso, o persona joven con cuadro isquémico, ya que el rápido inicio de corticoides o terapia inmunomoduladora, puede dar buena respuesta y evitar el daño endotelial crónico.

\section{Conflictos de interés}

Los autores declaran no tener de manera directa o indirecta, ningún tipo de conflicto de intereses financieros, académicos o laborales que puedan poner en peligro la validez de este estudio.

\section{Financiación}

Este trabajo se realizó con el apoyo financiero propio.

\section{Referencias}

1. Numano F, Okawara M, et al. Takayasu's arteritis. Lancet 2000;356:1023-25.

2. Matsuyama A, Sakai N. Ishigami M, Hircaka H, Kashine S, Hirata A, et al., Matrix metalloproteinases as novel disease markers in Takayasu arteritis. Circulation;2003;108:14691473.

3. Caccamise WC, Whitman JF. Pulse-less disease; a preliminary case report. Am Heart J; 44 (4):628-33.

4. Harrison, Braunwald E, Kasper D, Fauci A, Longo D, Hauser $\mathrm{S}$, et al.Harrison Principios de Medicina Interna. Mc Graw Hill, vol II. 18 Edición, 2012; p. 2065.

5. Savage C, Harper L, Cockwell P, Adu D, Howie AJ. ABC of arterial and vascular disease: vasculitis. British Med. Journal. 2000;520:1325-1328

6. Sato EI, Lima DNX, et al. Takayasu's arteritis treatment and prognosis. International Journal of Cardiology 2000; 75:163166.

7. Cañas CA, Jimenez CA, Ramirez LA, Uribe O, Tobón I, Torrenegra A, et al. Takayasu in Colombia. Int $\mathrm{J}$ Cardiol
1998;66:73-9.

8. Ochoa C, Ramírez F, Quintana G, Toro C, Cañas C; Osio LF, et al. Epidemiología de las vasculitis primarias en Colombia y su relación con lo informado para Latinoamérica / Epidemiology of primary vasculitis in Colombia and its relation with reported for latin america Rev.Colomb.Reumatol. 2009;16(3):248-263

9. Raghavan R, Krishnamurthy S, Ganesh RN, Kumar RR, Mahadevan S. Takayasu arteritis: Association with mesangioproliferative glomerulonephritis in a 9-year-old child. Indian J Nephrol. 2014;24(6):407-408.

10. Korzets Z, Barenboim E, Bernheim J, Mekori Y, Bernheim J. Mesangioproliferative glomerulonephritis, antiphospholipid antibodies, and Takayasu's arteritis. Nephrol Dial Transplant. 1998;13:991-3

11. Zilleruelo GE, Ferrer P, Garcia OL, Moore M, Pardo V, Strauss J. Takayasu's arteritis associated with glomerulonephritis. A case report. Am J Dis Child. 1978;132:1009-13.

12. BOUBAKAR K, Kaaroud H, Goucha R, Kheder A. Lesión renal en la arteritis de Takayasu Artículo francés Nephrol Ther. 2014;10(6):451-6.http://doi:10.1016/j.nephro. 2014.07.483. Epub 2014 Oct 23.

13. Johnston SL, Lock RJ, Gompels MM. Takayasu arteritis: a review. J Clin Pathol. 2002;55:481-486.

14. Sato EI, Lima DNX, et al. Takayasu's arteritis treatment and prognosis. International Journal of Cardiology 2000; 75 163166.

15. Noris M, Daina E, Gamba S, Bonazzola S, Remuzzi G. Interleukin- 6 and Rantes in Takayasu arteritis: a guide for therapeutic decisions?. Circulation 1999;100:155.

16. Stone J. Vasculitis: A Collection of Pearls and Myths. Rheum Dis Clin N Am 2007; 33:691-739.

17. Vega-Cornejo G, Meza-Beltrán J. Arteritis de Takayasu en el recién nacido, un diagnostico que sospechar. Reumatología clínica 2015;11 174-176.

18. Hernández-Rodríguez J, Espinosa G, Cid M.C, Cervera R Protocolo diagnóstico de la afectación neurológica central en conectivopatías y vasculitis. Medicine 2009;10:2091-5.

19. Babyn P, Doria A. Radiologic investigation of rheumatic disease. Pediatric Clin North Am. 2005;52:373-411.

20. Savage C, Harper L, Cockwell P, Adu D, Howie AJ. ABC of arterial and vascular disease: vasculitis. British Med. Journal. 2000;520:1325-1328.

21. Arend W, Michel B, Bloch D, Hunder G, Calabrese L, Edworthy S, et al. The American College of Rheumatology 1990 criteria for the classification of Takayasu arteritis. Arthritis Rheum 1990; 33(8):1129-1134.

22. Ishikawa K. Diagnostic approach and proposed criteria for the clinical diagnosis of Takayasu's arteriopathy. J Am Coll Cardiol 1988;12(4):964-972.

23. Gormezano N, Dos Santos M.C, Mikito O, Hulle C, Sachetti B. Associacão entre febre reumática e arterite de Takayasu. Relato de caso. Rev Brasileira de Reumatología. http://dx.doi.org/10.1016/j.rbr.2014.09.001.

24. Alibaz-Oner F, Sibel Z, Haner D. Advances in the diagnosis, assessment and outcome of Takayasu's arteritis. Clin Rheum. 2012;32:541-630

25. Ruige JB, Van Geet C, Nevelsteen A: A 16-year survey of Takayasu's Arteritis in a tertiary Belgian centre. Int Angiol 2003;22:414-420.

26. Kerr GS, Hallahan CW, Giordano J, Leavitt RY, Faucitt AS: Takayasu's Arteritis. Ann Int Med 1994;120:919-929.

27. Park MC, Lee SW, Park YB, Chung NS, Lee SK: Clinical characteristics and outcomes of Takayasu's Arteritis: analysis of 108 patients using standardized criteria for diagnosis, activity assessment and angiographic classification. Scand J Rheumatol, 2005;34:284-292.

28. Savage C, Harper L, Adu D. Primary Systemic Vasculitis. Lancet. 1997;349:553-558. 